Acta Biologica Sibirica

Journal of Biology

Founded in 2015
Altai State University

www.asu.ru

ISSN 2412-1908

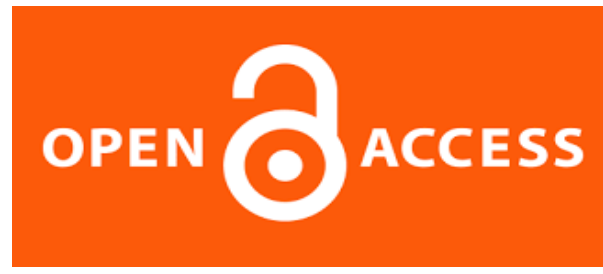

Acta Biologica Sibirica, 2017, 3(4), 99-112

\title{
New records of long-legged flies (Diptera, Dolichopodidae) from Central and North-Eastern Iran
}

\author{
I.Ya. Grichanov ${ }^{1}$, A. Ahmadi ${ }^{2}$, O.E. Kosterin ${ }^{3}$ \\ ${ }^{1}$ All-Russian Institute of Plant Protection, Podbelskogo 3, 196608, St.Petersburg-Pushkin, Russia. \\ ${ }^{2}$ Baran Plant Protection Institute, Arak, Markazi Province, Iran. \\ ${ }^{3}$ Institute of Cytology \& Genetics SB RAS, Acad. Lavrentyev ave. 10, Novosibirsk, 630090, Russia; Novosibirsk \\ State University, Pirogova str. 2, Novosibirsk, 630090, Russia; \\ E-mail: grichanov@mail.ru; aezam.ahmadi@gmail.com; kosterin@bionet.nsc.ru
}

During a 2016-2017 survey conducted in Isfahan, Lorestan, Markazi, North Khorasan, Razavi Khorasan, South Khorasan and Tehran provinces located in the Central and North-Eastern Iran, about 1000 specimens of Dolichopodidae were collected and identified. Eight dolichopodid species [Dolichopus jaxarticus Stackelberg, 1927, Hydrophorus viridis (Meigen, 1824), Medetera diadema (Linnaeus, 1767), M. lamprostoma Loew, 1871, M. roghii Rampini et Canzoneri, 1979, Tachytrechus kowarzi Mik, 1864, Tachytrechus sogdianus Loew, 1871, and Thinophilus flavipalpis (Zetterstedt, 1843)] are recorded for the first time in Iran. Dolichopus subimmaculatus Kazerani, Pollet, Khaghaninia, 2017, is placed in synonymy with Dolichopus perversus Loew, 1871 (syn. nov.). Lectotype is designated for D. perversus.

Key words: Dolichopodidae; Iran; Isfahan; Lorestan; Markazi; North Khorasan; Razavi Khorasan; South Khorasan; Tehran; new records

\section{Introduction}

Approximately 8000 (including about 100 fossil) described species and 288 (including 30 fossil) genera of the longlegged flies (Dolichopodidae) are known in the world fauna (Grichanov, 2017). The most recent Iranian list of these flies includes 114 species collected mainly from the north-west of Iran (Grichanov, 2016). Later Medetera abstrusa Thuneberg, 1955, M. belgica Parent, 1936, M. freyi Thuneber, 1955, M. feminina Negrobov, 1967 and M. pallipes (Zetterstedt, 1843) from the Guilan and Mazandaran provinces (Kazerani et al., 2016a), and Sciapus longulus (Fallén, 1823) from the Ardabil province (Tajmiri et al., 2016) were reported at the $22^{\text {nd }}$ Iranian Plant Protection Congress without indicating original material. Grichanov, Ahmadi (2016b) described a new species Poecilobothrus lorestanicus from the Lorestan province, and Grichanov, Ahmadi (2017a) described a new species Lamprochromus occidasiaticus from Turkey and the Markazi province of Iran. Ahmadi et al. (2017) recorded for the first time in Iran Chrysotus pennatus Lichtwardt, 1902, Sybistroma impar (Rondani, 1843), Tachytrechus beckeri Lichtwardt, 1917, Campsicnemus armatus (Zetterstedt, 1849), C. magius (Loew, 1845), C. tomkovichi Grichanov, 2009, Syntormon fuscipes (von Roser, 1840) and the genus Micromorphus Mik, 1878, collected in the Markazi and Lorestan provinces. The presence of Chrysotus obscuripes Zetterstedt, 1838 and Medetera pallipes in Iran was confirmed, and the presence of Dolichopus excisus Loew, 1859, in Iran was questioned.

Recently, Kazerani et al. (2017) have described eight new for science species, i.e. Dolichopus fuscicercus, D. subimmaculatus, Gymnopternus atratus, G. flavitibia, Hercostomus albicoxa, H. setitibia, Poecilobothrus annulitarsis and P. innotabilis from the north-west of Iran (East Azerbaijan, West Azerbaijan, Ardabil, Guilan and Mazandaran provincies). The 
Grichanov, I.Ya. et al. (2017). New records of long-legged.... Acta Biologica Sibirica, 2017, 3(4), 99-112

authors have also excluded Dolichopus plumipes (Scopoli, 1763), D. immaculatus Becker, 1909, Gymnopternus angustifrons (Staeger, 1842), Poecilobothrus chrysozygos (Wiedemann, 1817) and P. bigoti Mik, 1883, from the Iranian fauna.

Information on Dolichopodidae of the Central Iranian provinces has not been available before our investigations. Grichanov et al. (2010), Grichanov, Ahmadi (2016b, 2017a) and Ahmadi et al. (2016, 2017) published original data on species collected in the Markazi and Lorestan provinces. None species was reported from the Isfahan province. Becker, Stein (1913), who treated the material collected by N. Zarudny (St. Petersburg, Russia) during 1898 and 1901 expeditions, mentioned two species of long-legged flies from "Khorasan". Dolichopus notatus Staeger, 1842 (as Dolichopus notabilis Zetterstedt, 1843) was collected at "Kerat, Landschaft Haschtadan", now in the Razavi Khorasan province $\left(34.57^{\circ} \mathrm{N}\right.$, $60.55^{\circ} \mathrm{E}$ ). Syntormon pallipes (Fabricius, 1794) was found at the locality "Gerri-schatur", probably in the same province. Grichanov (Grichanov et al., 2010; Grichanov, Negrobov, 2014; Grichanov, Ahmadi, 2016a) treated also the material (in the Zoological Museum of Moscow State University, Moscow, Russia) collected by the Russian diplomat N.N. Filippov (under the pseudonym Zhenzhurist) in 1936-1938 at Tehran and its environs at Rasht, Jarjarud, "Shimran" (=Shemiran), "Pahlevi" (=Bandar-e Anzali), and 14 species were identified, including a new species Sciapus iranicus Grichanov \& Negrobov, 2014.

\section{Material and methods}

During 2017 season, about 1000 specimens have been sampled by authors of this paper mostly by standard sweep net. Some specimens (e.g., Medetera spp.) have been collected by use of a specimen jar. In addition, a small collection of dolichopodid flies sampled in 2016 was received from the Khorasan Plant Protection Station. Most of the identified species are included in the keys to more than 500 Dolichopodidae species of the Caucasus and Eastern Mediterranean (Grichanov, 2007). The general species distribution is given after Negrobov et al. (2013) and Grichanov (2017). Type localities are provided, and country lists (in Asia) are arranged alphabetically. The collectors of specimens were the first two authors of this paper unless otherwise is noted. Photos of some species newly recorded from Iran and habitat photos are provided. The specimens have been studied with a ${ }^{\circledR}$ Zeiss Discovery V-12 stereomicroscope and ${ }^{\circledR}$ AxioCam MRc5 camera attachment. They will be deposited in collections of the Zoological Institute of the Russian Academy of Sciences, St. Petersburg (ZIN), and the Zoological Museum of Moscow State University, Moscow, Russia (ZMMU).

References are given for species reported formerly from the Lorestan, Markazi, Razavi Khorasan and Tehran provinces of Iran. For the complete Iranian reference list see Grichanov (2016).

\section{New records of Dolichopodidae}

\section{Argyra Macquart, 1834}

Argyra leucocephala (Meigen, 1824)

REFERENCES. Grichanov et al., 2010: 196 (Tehran); Ahmadi et al., 2016: 192 (Lorestan); 2017: 65 (Lorestan).

MATERIAL. Lorestan prov.: 7 km NE Borujerd, Hayan village env., 3347'27-48'14" N, 4854'16"-56'31" E, 1688-1858 m a.s.l., 25.V.2017, 1 ऊิ.

DISTRIBUTION. Type locality: not given [Europe]. Asia (Iran, Israel, Turkey), Caucasus (Azerbaijan, Russia: Adygea, Chechnya, Krasnodar Territory), Europe, North Africa.

\section{Campsicnemus Haliday, 1851}

\section{Campsicnemus armatus (Zetterstedt, 1849)}

REFERENCES. Ahmadi et al., 2017: 68 (Lorestan).

MATERIAL. Markazi prov.: Arak env., 26 km W, Baneh village, 3405' N, 49²4' E, 2117 m a.s.l., 19.V.2017, 20'; Shazand

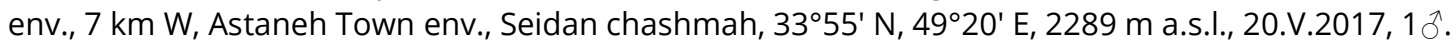

DISTRIBUTION. Type locality: Denmark: Rosenthal, Gryphium. Asia (Iran, Mongolia, Russian Far East, Siberia, Turkey), Europe, North and Tropical Africa. New species for Markazi Province.

\section{Campsicnemus curvipes (Fallén, 1823)}

REFERENCES. Ahmadi et al., 2017: 69 (Markazi, Lorestan).

MATERIAL. Lorestan prov.: 22 km S Aligudarz, Golbahar River at Golbahar-e Seikh Miri and Golbahar-e Mohammad

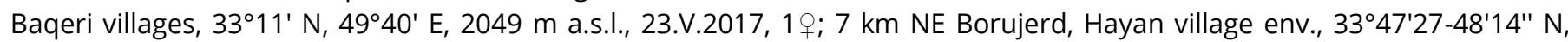

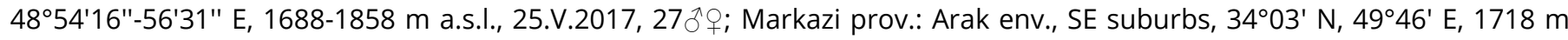

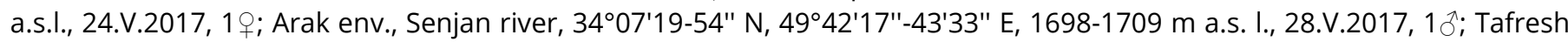

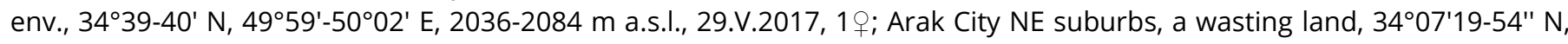
4942'17"-43'33" E, 1698-1709 m a.s.l., 28.V.2017, O. Kosterin, 1 q (in alcohol).

DISTRIBUTION. Type locality: not given [Europe]. Asia (Iran, Siberia, Turkey), Caucasus (Abkhazia, Armenia, Azerbaijan, Russia: Adygea, Alania, Chechnya, Dagestan, Kabardino-Balkaria, Karachai-Cherkessia, Krasnodar Territory, Stavropol Territory), Europe, North Africa.

Campsicnemus tomkovichi Grichanov, 2009

REFERENCES. Ahmadi et al., 2017: 69 (Markazi).

MATERIAL. Markazi prov.: 8 km SE Arak, Baghdadi, 3401' N, 4945' E, 1974 m a.s.l., 18.V.2017, $10^{\text {; }}$; Arak env., 26 km W,

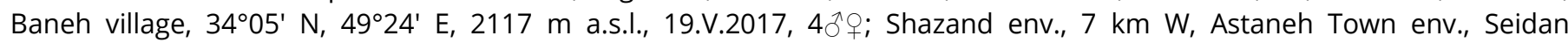
chashmah, $33^{\circ} 55^{\prime} \mathrm{N}, 49^{\circ} 20^{\prime} \mathrm{E}, 2289 \mathrm{~m}$ a.s.l., 20.V.2017, $60^{\wedge}$ \%. 
Grichanov, I.Ya. et al. (2017). New records of long-legged.... Acta Biologica Sibirica, 2017, 3(4), 99-112

DISTRIBUTION. Type locality: Azerbaijan: Yardimli [district], Kreki. Asia (Iran), Caucasus (Armenia, Azerbaijan).

\section{Campsicnemus umbripennis Loew}

REFERENCES. Ahmadi et al., 2016: 192 (Markazi); 2017: 69 (Markazi).

MATERIAL. Lorestan prov.: $41 \mathrm{~km}$ S Aligudarz, Kagelestan-e Bar Aftab village, 3302' N, 49³9' E, $1841 \mathrm{~m}$ a.s.l.,

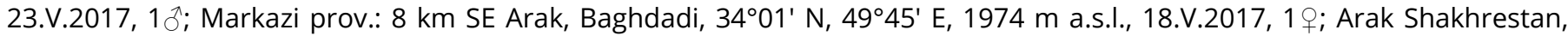
Masumiyeh rural district, env. of Baghdadi and Hosseinabad villages, 3401'28-56" N, 4945'12-46'21" E, 1955-2015 m a.s.l.,

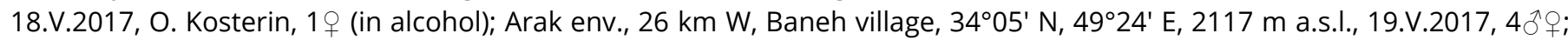

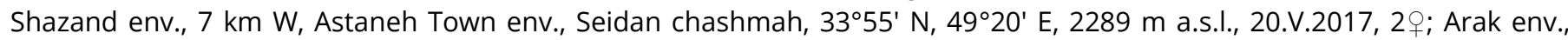

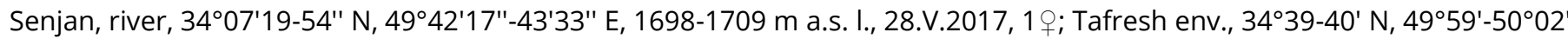
E, 2036-2084 m a.s.l., 29.V.2017, 13;

DISTRIBUTION. Type locality: Austria. Asia (Afghanistan, Iran, Iraq, Israel, Tajikistan, Turkey, Turkmenistan), Caucasus (Abkhazia, Armenia, Azerbaijan, Georgia, Russia: Adygea, Alania, Chechnya, Kabardino-Balkaria, Karachai-Cherkessia, Krasnodar Territory), Europe, North Africa. New for Lorestan province.

\section{Chrysotus Meigen, 1824}

\section{Chrysotus sp.}

MATERIAL. Razavi Khorasan prov.: Torqabeh, 23.VII.2016, Motamednia, 1 ( (in alcohol).

REMARK. First record of the genus from the Razavi Khorasan province.

\section{Chrysotus suavis Loew, 1857}

REFERENCES. Grichanov et al., 2010: 198 (Markazi); Ahmadi et al., 2016: 192 (Markazi); 2017: 66 (Lorestan).

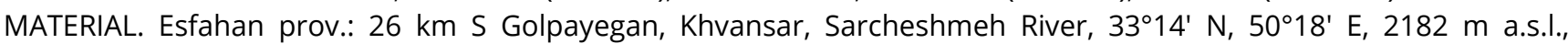
21.V.2017, 19; Lorestan prov.: 22 km S Aligudarz, Golbahar River at Golbahar-e Seikh Miri and Golbahar-e Mohammad Baqeri villages, $33^{\circ} 11^{\prime} \mathrm{N}, 49^{\circ} 40^{\prime} \mathrm{E}, 2049 \mathrm{~m}$ a.s.l., 23.V.2017, 2 ; $19 \mathrm{~km}$ SW Dorud, Sezar River at $2 \mathrm{~km}$ downstream of Bisheh

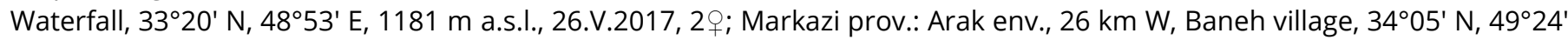
E, 2117 m a.s.l., 19.V.2017, 19; Garehchay River, 0.8 km SSW Gavigodar village, 3406'28-40" N, 49²1'45-57" E, 1803-1805 m

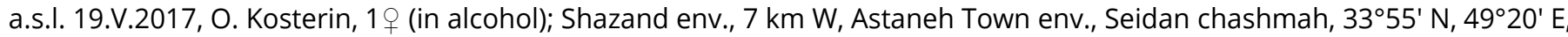
$2289 \mathrm{~m}$ a.s.l., 20.V.2017, 1 ; ; Arak City NE suburbs, a wasting land, 3407'19-54" N, 49²4'17"-43'33" E, 1698-1709 m a.s.l., 28.V.2017, O. Kosterin, $2 \widehat{\jmath}$ (in alcohol).

DISTRIBUTION. Type locality: Germany: "Coln"; Austria: "Neusiedler See in Ungarn". Asia (Afghanistan, Palaearctic China, Iran, Iraq, Israel, Kyrgyzstan, Middle Asia, Mongolia, Russian Far East, Siberia, Turkey), Caucasus (Armenia, Azerbaijan, Georgia, Russia: Adygea, Alania, Kabardino-Balkaria, Krasnodar Territory, Rostov Region), Europe, North Africa. New for Esfahan province.

\section{Dolichopus Latreille, 1796}

Dolichopus jaxarticus Stackelberg, 1927 (Figs. 1-2)

MATERIAL. Razavi Khorasan prov.: Mohamadia, 27.VIII.2016, Motamednia, 10,1 1 (in alcohol).

ADDITIONAL MATERIAL EXAMINED. South Tajikistan: Dusti env., 24.VI.1985, Grichanov \& Shamshev, $10^{\Uparrow}$.

DISTRIBUTION. Type locality: "prov. Syrdarjensis et Samarkandica (Tshardary)" [South Kazakhstan, Uzbekistan]. Asia

(China: Xinjiang; Kazakhstan, Uzbekistan), Ukraine (Kherson). New for Iran and Tajikistan.

REMARKS. Negrobov (1991) included in error South Russia in the distribution area of the species.

\section{Dolichopus perversus Loew, 1871}

=Dolichopus subimmaculatus Kazerani, Pollet, Khaghaninia, in: Kazerani et al., 2017: 117, syn. nov. Type locality: Iran: East Azerbaijan, Arasbaran, Chichekli.

=Dolichopus immaculatus of authors, not Becker, 1909. Pârvu, 1996: 280; Kazerani et al., 2014: 140; Khaghaninia et al., 2014: 589.

REFERENCES. Grichanov et al., 2010: 198 (Tehran); Ahmadi et al., 2017: 66 (Lorestan, Markazi).

MATERIAL. Esfahan prov.: Golpayegan Shahrestan, Ghomrood River left bank just upstream of the lower reserve, 3325'02-14" N, 5007'08-12" E, 1924-1931 m a.s.l., 21.V.2017, O. Kosterin, 1 ㅇ (in alcohol); Markazi prov.: Arak Shakhrestan, Masumiyeh rural district, env. of Baghdadi and Hosseinabad villages, 3401'28-56" N, 4945'12-46'21" E, 1955-2015 m a.s.l.,

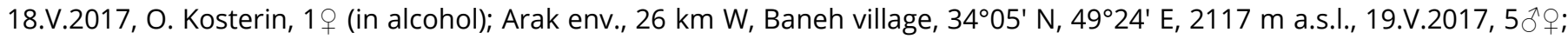

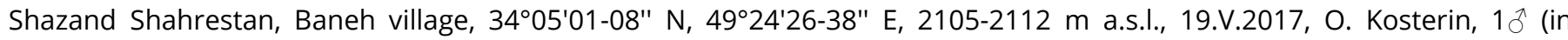
alcohol); Arak City NE suburbs, a wasting land, 3407'19-54" N, 4942'17"-43'33" E, 1698-1709 m a.s.l., 28.V.2017, O.

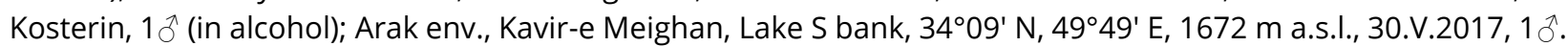

TYPE MATERIAL EXAMINED. Lectotype (here designated to fix the current taxonomic concept and ensure consistent

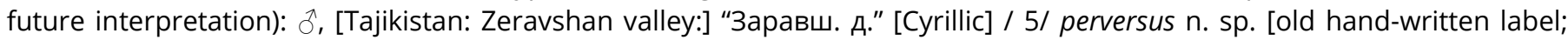
ZMMU]. Possible paralectotypes: $10 \hat{}$, 5 ㅇ, same locality [ZIN]; [Uzbekistan:] "Shakhimardan / 5" [Shohimardon exclave within Kyrgyzstan, $39.98^{\circ} \mathrm{N}, 71.80^{\circ} \mathrm{E}$ ], $1{ }^{\circ}$; [Kyrgyzstan: about $12 \mathrm{~km}$ from Osh:] Mody / 7, $1 \delta^{\circ}, 3$; ; [Uzbekistan:] Tashkent / 8, 13; [Tajikistan:] Magian / 6, 1 ; ; [Kyrgyzstan: Osh region:] Gulsha / 10, 1 ( (ZMMU, ZIN).

ADDITIONAL MATERIAL EXAMINED. Tajikistan: Sangvor, 10.VI.1977, Zlobin, 40̄; Turkey near Akseki, 1750 m, 29.V.2006, N. Vikhrev, 10 .

DISTRIBUTION. Type locality: Tajikistan: "Zarawschan Thal [=Zeravshan valley], Turkestan". Asia (Iran, Israel, Kazakhstan, Kyrgyzstan, Tajikistan, Turkey), Caucasus (Abkhazia, Armenia). New for Esfahan province. 
REMARKS. The detailed description and illustrations of Dolichopus subimmaculatus (Kazerani et al., 2017) have no morphological differences from the studied material of Dolichopus perversus from several countries, including possible syntypes of $D$. perversus found in the ZMMU and ZIN collections. The discoloration of the specimens collected by A.P. Fedchenko is related to about 150 years storing of the collection. A colour difference of femora noted by Kazerani et al. (2017) in their new species belongs to the intra-specific variation of $D$. perversus. Therefore, we consider the two names as synonyms.

\section{Dolichopus siculus Loew, 1859}

REFERENCES. Ahmadi et al., 2016: 192 (Markazi; as Dolichopus excisus Loew, 1859), 2017: 66 (Lorestan, Markazi).

MATERIAL. Markazi prov.: Shazand Shahrestan, Bolagh spring, 3359'14-17" N, 49²1'38-43" E, 1853-1861 m a.s.l.,

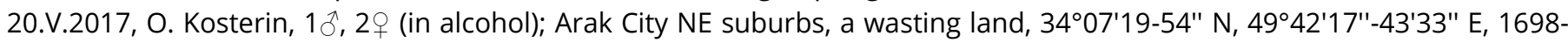
1709 m a.s.l., 28.V.2017, O. Kosterin, 1 i (in alcohol).

DISTRIBUTION. Type locality: Italy: Sidy. Asia (Golan Heights, Iran, Israel), Europe (Bulgaria, France, Italy).

\section{Dolichopus signifer Haliday, 1832}

REFERENCES. Grichanov et al., 2010: 198 (Markazi, Tehran); Ahmadi et al., 2016: 192 (Lorestan, Markazi), 2017: 66 (Lorestan, Markazi).

MATERIAL. Esfahan prov.: $15 \mathrm{~km}$ WWS Golpayegan, Ghomrood River left bank just upstream of the lower reserve, $33^{\circ} 25^{\prime} \mathrm{N}, 50^{\circ} 07^{\prime} \mathrm{E}, 1925 \mathrm{~m}$ a.s.l., 21.V.2017, 23; Golpayegan Shahrestan, Ghomrood River left bank just upstream of the

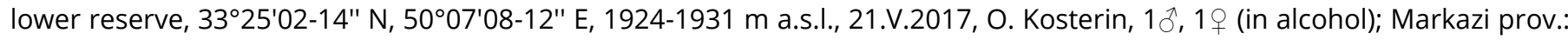

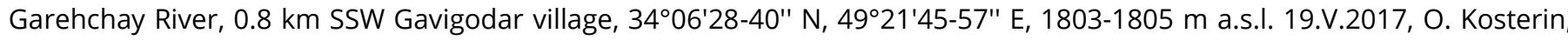
1 (in alcohol); Arak env., Senjan, river, 3407'19-54" N, 4942'17"-43'33" E, 1698-1709 m a.s. l., 28.V.2017, 2o ; Arak City NE

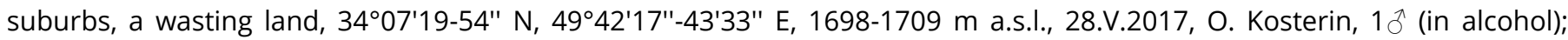
North Khorasan prov.: Golian, 23.VII.2016, Motamednia, Razavi Khorasan prov.: Chenaran, 22.VII.2016, Motamednia, 4ภ, 5; Node, 11.IX.2016, Motamednia; Torqabeh, 27.VII, 15.IX.2016, Motamednia, 20, 4 ㅇ (in alcohol).

DISTRIBUTION. Type locality: Ireland: Roundstone Bay. Asia (Afghanistan, Iran, Kazakhstan, Tajikistan, Turkey, Turkmenistan, Uzbekistan), Caucasus (Georgia, Russia: Chechnya, Kabardino-Balkaria, Krasnodar Territory, Rostov Region), Europe, North Africa. New for Esfahan, North Khorasan and Razavi Khorasan provinces.

\section{Hercostomus Loew, 1857}

\section{Hercostomus fulvicaudis (Haliday, 1851)}

2 .

MATERIAL. Markazi prov.: Arak env., Senjan, river, 3407'19-54" N, 49²4'17"-43'33" E, 1698-1709 m a.s. I., 28.V.2017,

DISTRIBUTION. Type locality: England. Asia (China, Iran, Tajikistan, Turkey, Turkmenistan), Europe. New for Markazi province.

\section{Hydrophorus Fallén, 1823}

Hydrophorus balticus (Meigen, 1824)

REFERENCES. Ahmadi et al., 2017: 68 (Markazi).

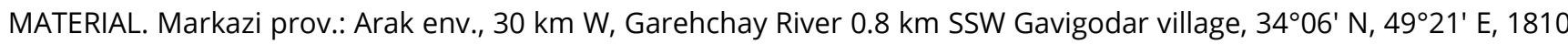
m a.s.l., 19.V.2017, 3수.

DISTRIBUTION. Type locality: Germany: Hamburg. Asia (Afghanistan, Algeria, Cyprus, Iran, Israel, Mongolia, Siberia, Turkey), Caucasus (Armenia, Azerbaijan, Georgia, Russia: Adygea, Alania, Chechnya, Kabardino-Balkaria, KarachaiCherkessia, Krasnodar Territory). Europe, North Africa.

\section{Hydrophorus praecox (Lehmann, 1822)}

REFERENCES. Grichanov et al., 2010: 199 (Tehran).

MATERIAL. Lorestan prov.: 7 km NE Borujerd, Hayan village env., 3347'27-48'14" N, 4854'16"-56'31" E, 1688-1858 m a.s.l., 25.V.2017, 7 $\hat{\text { o }}$; Aligudarz Shahrestan, Golbahar River at Golbahar-e Seikh Miri and Golbahar-e Mohammad Baqeri

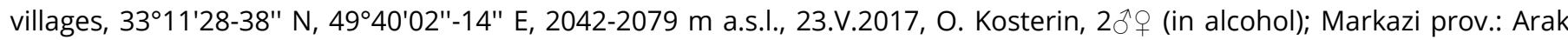

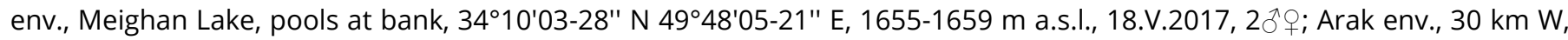

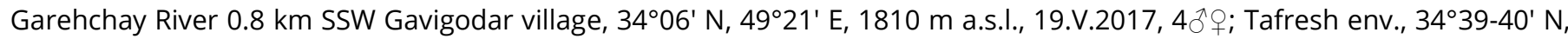

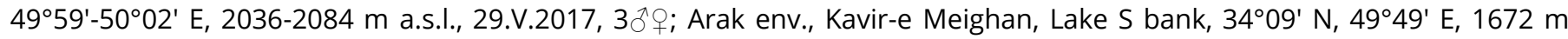

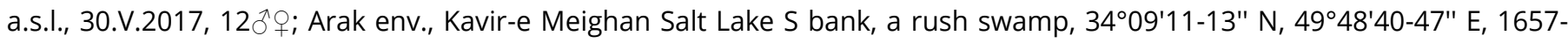
1659 m a.s.l., 30.V.2017, O. Kosterin, 5 ㅇ (in alcohol).

DISTRIBUTION. Type locality: Germany: Hamburg. Asia (China, Cyprus, Iraq, Iran, Israel, Kazakhstan, Mongolia, Turkey), Caucasus (Abkhazia, Georgia, Russia: Chechnya, Kabardino-Balkaria, Krasnodar Territory, Stavropol Territory), Europe, North Africa; Oriental, Australasian, Afrotropical and Neotropical Regions. New for Lorestan and Markazi provinces.

Hydrophorus viridis (Meigen, 1824) (Figs. 6-7)

MATERIAL. Esfahan prov.: $15 \mathrm{~km}$ WSW Golpayegan, Ghomrood River left bank just upstream of the lower reserve, $33^{\circ} 25^{\prime} \mathrm{N}, 50^{\circ} 07^{\prime} \mathrm{E}, 1925 \mathrm{~m}$ a.s.l., 21.V.2017, $10^{\text {’ }}$.

DISTRIBUTION. Type locality: Austria. Asia (Afghanistan, China, Kazakhstan, Mongolia, "Palestine", Tajikistan, Uzbekistan), Caucasus (Russia: Krasnodar Territory, Rostov Region), Europe, North Africa; Oriental China. New for Iran.

Liancalus Loew, 1857

Liancalus virens (Scopoli, 1763)

REFERENCES. Grichanov, Ahmadi, 2016a: 8 (Tehran). 
Grichanov, I.Ya. et al. (2017). New records of long-legged.... Acta Biologica Sibirica, 2017, 3(4), 99-112

MATERIAL. Lorestan prov.: $22 \mathrm{~km}$ S Aligudarz, Golbahar River at Golbahar-e Seikh Miri and Golbahar-e Mohammad

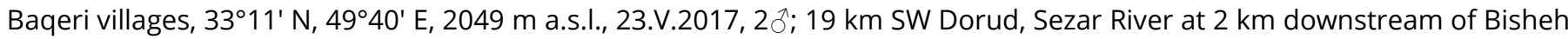

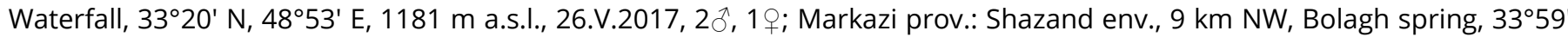

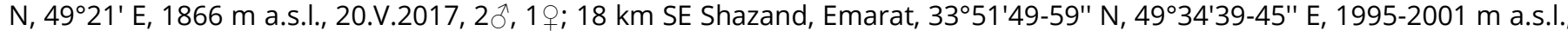

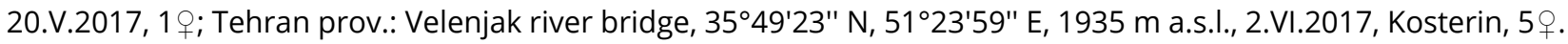

DISTRIBUTION. Type locality: not given ["Carnioliae indigena", Slovenia]. Asia (Cyprus, Iran, Israel, S Kazakhstan, Kyrgyzstan, "Siberia", Tajikistan, Turkey), Caucasus (Abkhazia, Azerbaijan, Georgia, Russia: Chechnya, Krasnodar Territory), Europe, North Africa. New for Lorestan and Markazi provinces.

REMARKS. Liancalus virens was found in the old Zhenzhurist's collection in the Zoological Museum of Moscow State University (Grichanov, Ahmadi, 2016a), being taken by the Russian diplomat and Diptera collector N.N. Filippov (under the pseudonym Zhenzhurist) more than 80 years ago in Tehran and here recorded from Iran for the second time. The species is rediscovered in northern environs of Tehran, and its local population survival conditions are probably favourable. The species has been also found in the Lorestan and Markazi provinces, being probably widespread in mountains of Iran. Adults are commonly found resting on vertical wet rock or concrete surfaces at springs and waterfalls.

\section{Medetera Fischer von Waldheim, 1819}

Medetera anjudanica Grichanov et Ahmadi, 2017

MATERIAL. Markazi prov.: Arak env., 35 km ESE, Anjudan village, [3359'03" N, 5002'01" E, 2036 m a.s.l., on house wall], 22.V.2017, 20.

REMARK. This species is described and illustrated separately (Grichanov, Ahmadi, 2017b).

Medetera diadema (Linnaeus, 1767) (Fig. 5)

MATERIAL. Tehran prov.: Velenjak, Inst. PI. Prot., $1 \hat{\jmath}, 20$.

DISTRIBUTION. Type locality: Sweden: Scania. Asia (Kazakhstan, Turkey, Siberia), Caucasus (Armenia, Azerbaijan, Georgia, Russia: Alania, Chechnya, Kabardino-Balkaria, Krasnodar Territory, Rostov Region, Stavropol Territory), Europe, North Africa. New for Iran.

REMARKS. Medetera diadema together with two more Medetera species (see below) was taken from Platanus tree trunks in a park at the Institute of Plant Protection in Tehran.

\section{Medetera lamprostoma Loew, 1871 (Figs. 3-4)}

MATERIAL. South Khorasan prov.: Mazarkahi, 5.IX.2016, Motamednia, 1 रे (in alcohol).

DISTRIBUTION. Type locality: Tajikistan: "Saravshan" [=Zerafshan] valley. Asia (Tajikistan, Turkmenistan, Uzbekistan). New for Iran.

\section{Medetera meridionalis Negrobov, 1967}

REFERENCES. Ahmadi et al., 2017: 68 (Lorestan, Markazi).

MATERIAL. Lorestan prov.: 20 km SE Borujerd, Hayan village env., 3347'27-48'14" N, 4854'16"-56'31" E, 1688-1858 m

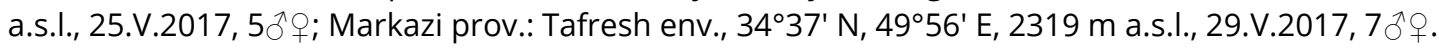

DISTRIBUTION. Type locality: Russia: Voronezh, near Novokhopersk river. Asia (Iran, Kazakhstan, Siberia, Turkey), Caucasus (Armenia, Azerbaijan, Georgia, Russia: Krasnodar Territory, Rostov Region). Europe.

Medetera pallipes (Zetterstedt, 1843)

REFERENCES. Ahmadi et al., 2017: 68 (Markazi).

MATERIAL. Esfahan prov.: $15 \mathrm{~km}$ WWS Golpayegan, Ghomrood River left bank just upstream of the lower reserve,

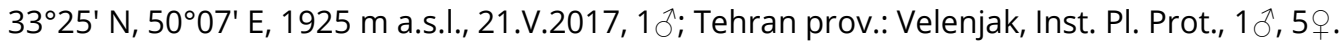

DISTRIBUTION. Type locality: Scania, "in Ostrog ad Wadstena; Botnia orientali ad Johannis Ro prope Torneä" [Sweden; Denmark]. Asia (Iran, Israel, Turkey), Caucasus (Georgia, Russia: Chechnya, Kabardino-Balkaria, Krasnodar Territory, Stavropol Territory), Europe, North Africa. New for Esfahan and Tehran provinces.

Medetera roghii Rampini et Canzoneri, 1979

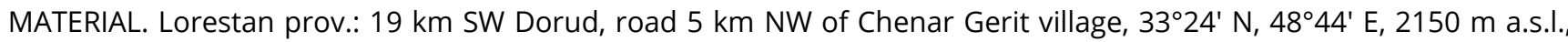
26.V.2017, 30र.

DISTRIBUTION. Type locality: Spain: Menorca. Italy (Sicilia), Malta and Spain (Menorca). New for Asia and Iran.

REMARKS. In contrast to closely related $M$. truncorum (see below), which is a common inhabitant of tree trunks, $M$. roghii has been collected from stones situated on rather dry mountain slope covered with sparse shrubs and xerophilous grasses. The species can be reliably distinguished from other related species by the morphology of hypopygium only; i.e. by the presence of a pair of long flattened apical setae on ventral lobe of surstylus and flattened epandrial setae (see Negrobov, Naglis, 2016).

\section{Medetera truncorum Meigen, 1824}

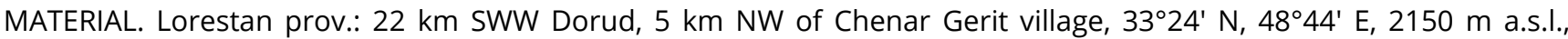

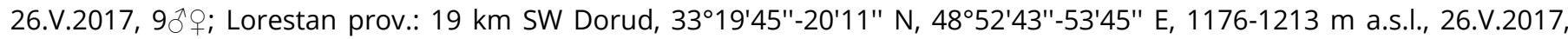

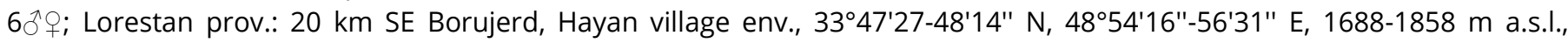
25.V.2017, 3ํำ; Razavi Khorasan prov.: Torqabeh, 27.VII.2016, Motamednia, $1 \hat{\jmath}$ (in alcohol); Tehran prov.: Velenjak, Inst. PI. Prot., 1 +

DISTRIBUTION. Type locality: Germany: Hamburg. Asia (Iran, Israel, Siberia, Turkey), Caucasus (Azerbaijan, Russia: Chechnya, Krasnodar Territory), Europe, North Africa; Nearctic Region. New for Lorestan, Razavi Khorasan and Tehran provinces. 


\section{Poecilobothrus Mik, 1878}

Poecilobothrus regalis (Meigen, 1824)

REFERENCES. Grichanov et al., 2010: 198 (Markazi); Ahmadi et al., 2017: 66 (Lorestan, Markazi).

MATERIAL. Lorestan prov.: 22 km S Aligudarz, Golbahar River at Golbahar-e Seikh Miri and Golbahar-e Mohammad

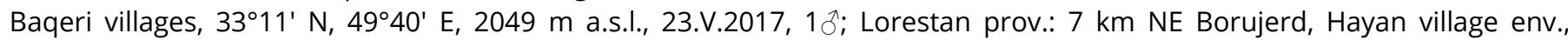

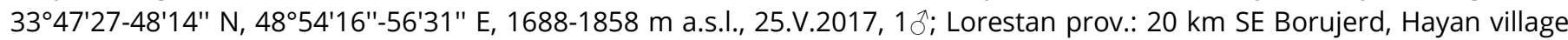

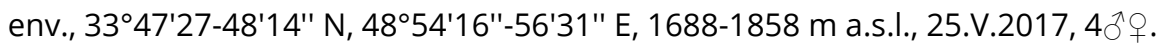

DISTRIBUTION. Type locality: not given. Asia (Iran, Turkey, Uzbekistan), Caucasus (Azerbaijan, Georgia, Russia: Chechnya, Kabardino-Balkaria, Krasnodar Territory, Rostov Region, Stavropol Territory). Europe.

\section{Rhaphium Meigen, 1803}

Rhaphium sp.

MATERIAL. Esfahan prov.: Golpayegan Shahrestan, Ghomrood River left bank just upstream of the lower reserve, 33²5'02-14" N, 5007'08-12" E, 1924-1931 m a.s.l., 21.V.2017, O. Kosterin, 1 q (in alcohol).

REMARKs. First record of the genus for Esfahan province. The species belongs to the Rhaphium brevicorne group.

\section{Rhaphium appendiculatum Zetterstedt, 1849}

REFERENCES. Ahmadi et al., 2016: 193 (Lorestan, Markazi), 2017: 68 (Lorestan).

MATERIAL. Lorestan prov.: 22 km S Aligudarz, Golbahar River at Golbahar-e Seikh Miri and Golbahar-e Mohammad

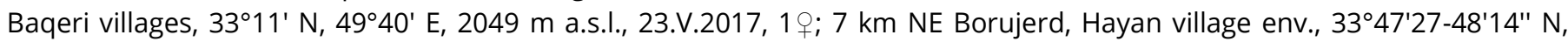
4854'16"-56'31" E, 1688-1858 m a.s.I., 25.V.2017, 1 ; ; 20 km SE Borujerd, Hayan village env., 3347'27-48'14" N, 4854'16"-

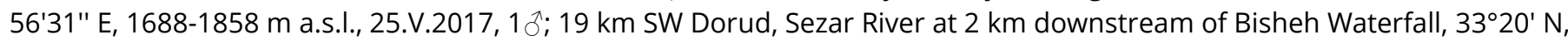

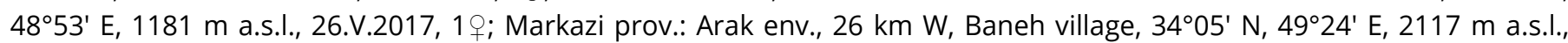

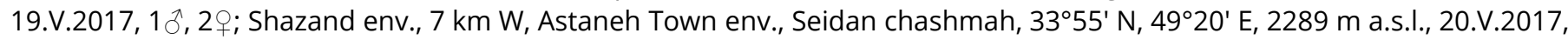

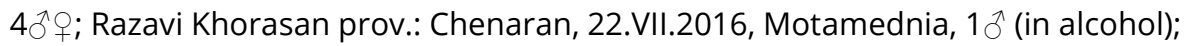

DISTRIBUTION. Type locality: Sweden: "Scania ad Esperod". Asia (Afghanistan, Iran, Turkey, "Middle Asia”), Caucasus (Abkhazia, Georgia, Russia: Adygea, Alania, Krasnodar Territory), Europe, North Africa. New for Razavi Khorasan province.

\section{Rhaphium brevicorne Curtis, 1835}

MATERIAL. Lorestan prov.: 7 km NE Borujerd, Hayan village env., 3347'27-48'14" N, 4854'16"-56'31" E, 1688-1858 m

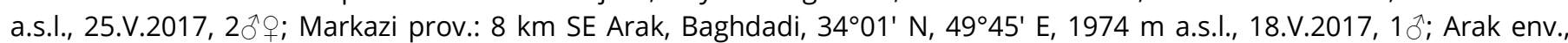
Senjan, river, 3407'19-54" N, 4942'17"-43'33" E, 1698-1709 m a.s. I., 28.V.2017, 1 ; ; Arak City NE suburbs, a wasting land, 3407'19-54" N, 4942'17"-43'33" E, 1698-1709 m a.s.l., 28.V.2017, O. Kosterin, $10^{\Uparrow}$ (in alcohol).

DISTRIBUTION. Type locality: England: Isle of Wight. Asia (Iran, Iraq, Israel, Lebanon, Tajikistan, Turkey), Caucasus (Russia: Krasnodar Territory), Europe, North Africa. New for Lorestan and Markazi provinces.

REMARKS. Kazerani et al. (2016b) mentioned this species from the north-west of Iran without indicating original material.

\section{Rhaphium micans (Meigen, 1824)}

REFERENCES. Grichanov et al., 2010: 198 (Markazi).

MATERIAL. Markazi prov.: Shazand Shahrestan, Bolagh spring, 3359'14-17" N, 49²1'38-43" E, $1853-1861 \mathrm{~m}$ a.s.l., 20.V.2017, O. Kosterin, 1§خ; Razavi Khorasan prov.: Torqabeh, 27.VII.2016, Motamednia, $1 \delta^{\Uparrow}$ (in alcohol).

DISTRIBUTION. Type locality: Germany: Hamburg. Asia (Palaearctic China, Iran, Tajikistan, Turkey, Siberia, Russian Far East), Caucasus (Abkhazia, Azerbaijan, Russia: Adygea, Kabardino-Balkaria, Karachai-Cherkessia, Krasnodar Territory, Rostov Region), Europe. New for Razavi Khorasan province.

\section{Sciapus Zeller, 1842}

Sciapus sp.

MATERIAL. Lorestan prov.: 22 km S Aligudarz, Golbahar River at Golbahar-e Seikh Miri and Golbahar-e Mohammad Baqeri villages, 3311' N, 4940' E, 2049 m a.s.l., 23.V.2017, 1 \%.

REMARK. First record of the genus for Lorestan province.

\section{Sybistroma Meigen, 1824}

\section{Sybistroma nodicornis Meigen, 1824}

REFERENCES. Ahmadi et al., 2017: 68 (Lorestan, Markazi).

MATERIAL. Lorestan prov.: 22 km S Aligudarz, Golbahar River at Golbahar-e Seikh Miri and Golbahar-e Mohammad

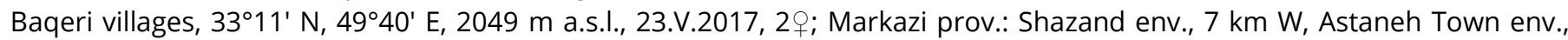

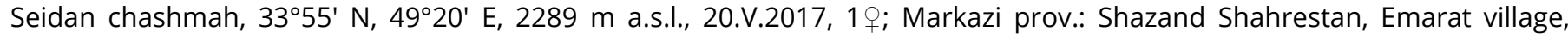
20.05.2015, 3351'49-59" N, 49³4'39-45" E, 1995-2001 m a.s.l., O. Kosterin, $1 \delta^{\curvearrowright}$ (in alcohol).

DISTRIBUTION. Type locality: not given. Asia (Iran, Iraq, Turkey), Europe, North Africa.

\section{Sympycnus Loew, 1857}

\section{Sympycnus pulicarius (Fallén, 1823)}

REFERENCES. Ahmadi et al., 2016: 194 (Markazi), 2017: 69 (Lorestan).

MATERIAL. Esfahan prov.: 26 km S Golpayegan, Khvansar, Sarcheshmeh River, 33¹4' N, $50^{\circ} 18^{\prime}$ E, 2182 m a.s.l.,

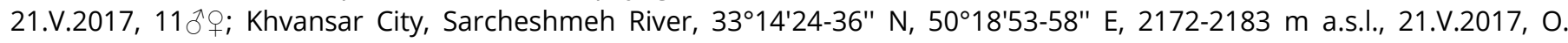
Kosterin, 1 + (in alcohol); Markazi prov.: Arak env., 26 km W, Baneh village, 3405' N, 49²4' E, 2117 m a.s.l., 19.V.2017, 1 ․ 
Grichanov, I.Ya. et al. (2017). New records of long-legged.... Acta Biologica Sibirica, 2017, 3(4), 99-112

DISTRIBUTION. Type locality: not given [Sweden]. Asia (Iran, Kazakhstan, Turkey, Siberia), Caucasus (Azerbaijan, Russia: Alania, Kabardino-Balkaria, Karachai-Cherkessia, Stavropol Territory), Europe; Nearctic Region. New for Esfahan province.

\section{Syntormon Loew, 1857}

\section{Syntormon denticulatus (Zetterstedt, 1843)}

REFERENCES. Ahmadi et al., 2016: 194 (Lorestan, Markazi), 2017: 69 (Markazi).

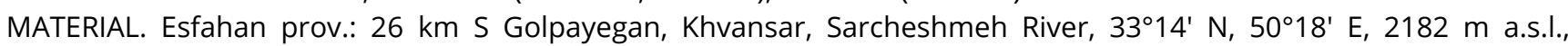
21.V.2017, 19; Lorestan prov.: 22 km S Aligudarz, Golbahar River at Golbahar-e Seikh Miri and Golbahar-e Mohammad

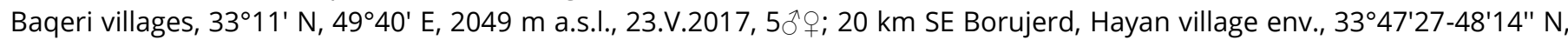
4854'16"-56'31" E, 1688-1858 m a.s.l., 25.V.2017, 20ํㅜ Markazi prov.: 8 km SE Arak, Baghdadi, 3401' N, 4945' E, 1974 m

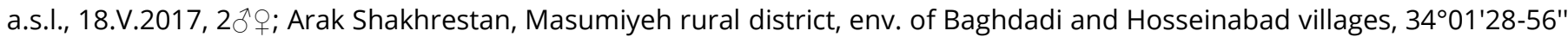

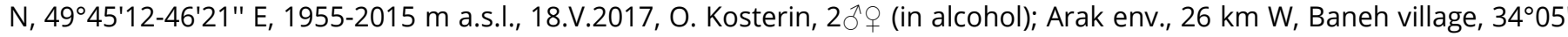
N, 49²4' E, 2117 m a.s.l., 19.V.2017, 1 ..

DISTRIBUTION. Type locality: Sweden: “Scania”. Asia (Afghanistan, Iran, Israel, Kyrgyzstan, Tajikistan, Turkey), Caucasus (Abkhazia, Armenia, Azerbaijan, Russia: Adygea, N Ossetia-Alania, Kabardino-Balkaria, Stavropol Territory), Europe, "North Africa". New for Esfahan province.

\section{Syntormon pallipes (Fabricius, 1794)}

REFERENCES. Becker, Stein, 1913: 597 (Khorasan); Grichanov et al., 2010: 201 (Markazi, Tehran); Ahmadi et al., 2016: 194 (Lorestan, Markazi), 2017: 69 (Lorestan, Markazi).

MATERIAL. Esfahan prov.: 26 km S Golpayegan, Khvansar, Sarcheshmeh River, 3314'32" N, 50¹8'56" E, 2182 m a.s.l., 21.V.2017, 2\%; Lorestan prov.: 22 km S Aligudarz, Golbahar River at Golbahar-e Seikh Miri and Golbahar-e Mohammad

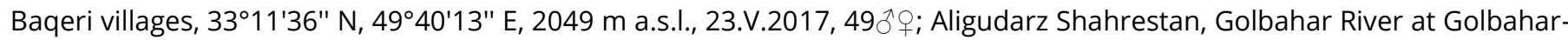
e Seikh Miri and Golbahar-e Mohammad Baqeri villages, 33¹1'28-38" N, 4940'02"-14" E, 2042-2079 m a.s.l., 23.V.2017, O.

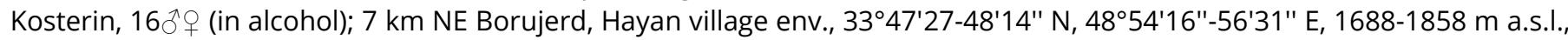

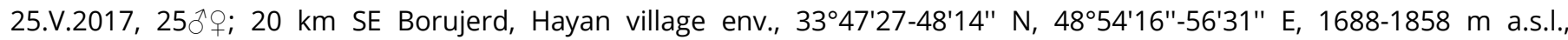

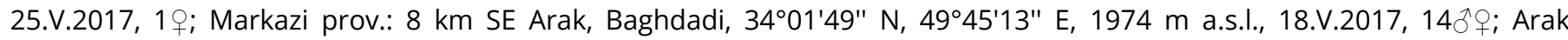

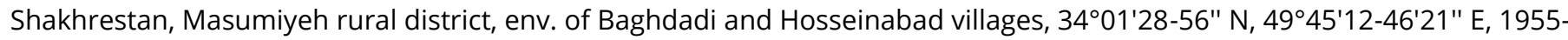

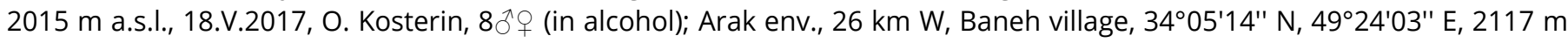
a.s.l., 19.V.2017, $100^{\prime}$ \%; Garehchay River, 0.8 km SSW Gavigodar village, 3406'28-40" N, 49²1'45-57" E, 1803-1805 m a.s.l. 19.V.2017, O. Kosterin, $10^{\lambda}$ (in alcohol); Shazand Shahrestan, Baneh village, 3405'01-08" N, 49²4'26-38" E, 2105-2112 m

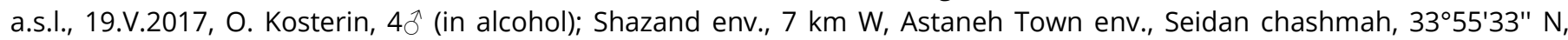
49²0'56" E, 2289 m a.s.l., 20.V.2017, Markazi prov.: Shazand Shahrestan, Emarat village, 3351'49-59" N, 49³4'39-45" E,

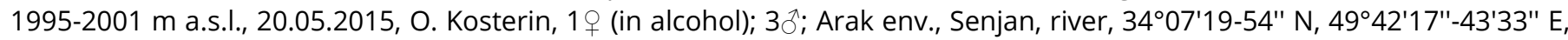

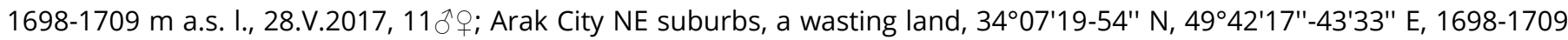

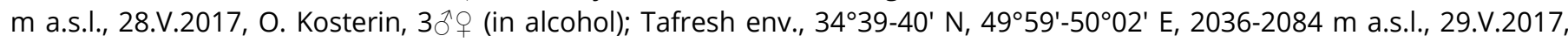

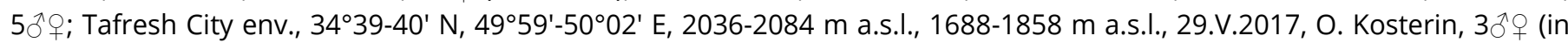
alcohol); Razavi Khorasan prov.: Chenaran, 22.VII.2016, Motamednia, 1ㅎ; Torqabeh, 23, 27.VII.2016, Motamednia, $2 \hat{\jmath}$ (in alcohol).

DISTRIBUTION. Type locality: Germany. Asia (Afghanistan, Palaearctic China, Iran, Iraq, Israel, Jordan, Kyrgyzstan, Tajikistan, Turkey, Uzbekistan), Caucasus (Abkhazia, Armenia, Azerbaijan, Georgia, Russia: Adygea, Alania, KabardinoBalkaria, Karachai-Cherkessia, Krasnodar Territory, Rostov Region), Europe, North Africa; Oriental China; Afrotropics. New for Esfahan province.

\section{Syntormon zelleri (Loew, 1850)}

REFERENCES. Grichanov et al., 2010: 201 (Tehran).

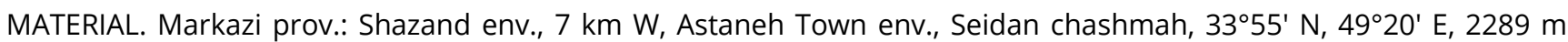
a.s.l., 20.V.2017, $2 \hat{\jmath}+$.

DISTRIBUTION. Type locality: Italy: “Sicilien”. Asia (Iran, Turkey, “Middle Asia”), Caucasus (Abkhazia, Azerbaijan, Russia: Chechnya, Krasnodar Territory), Europe. New for Markazi province.

\section{Tachytrechus Haliday, 1851}

\section{Tachytrechus kowarzi Mik, 1864 (Figs. 11-12)}

MATERIAL. Esfahan prov.: $15 \mathrm{~km}$ WWS Golpayegan, Ghomrood River left bank just upstream of the lower reserve, $33^{\circ} 25^{\prime} \mathrm{N}, 50^{\circ} 07^{\prime} \mathrm{E}, 1925 \mathrm{~m}$ a.s.l., 21.V.2017, $10^{2}$, 1\%; Markazi prov.: Arak env., $30 \mathrm{~km} \mathrm{~W}$, Garehchay River $0.8 \mathrm{~km}$ SSW

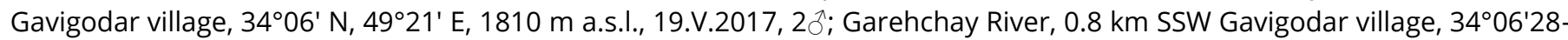
40" N, 49²1'45-57" E, 1803-1805 m a.s.l. 19.V.2017, O. Kosterin, 3ํㅜ (in alcohol).

DISTRIBUTION. Type locality: Hungary: Miskolcz, Oberungam. Asia (Turkey), Caucasus (Armenia, Azerbaijan), Europe. New for Iran.

\section{Tachytrechus notatus (Stannius, 1831)}

REFERENCES. Grichanov et al., 2010: 199 (Tehran); Ahmadi et al., 2017: 69 (Lorestan, Markazi).

MATERIAL. Lorestan prov.: $22 \mathrm{~km}$ S Aligudarz, Golbahar River at Golbahar-e Seikh Miri and Golbahar-e Mohammad

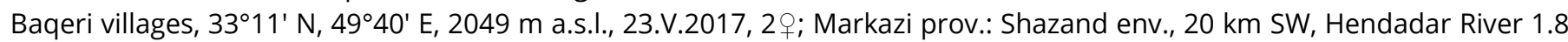

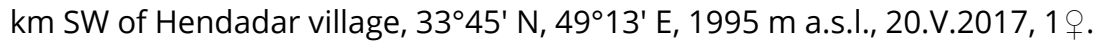


Grichanov, I.Ya. et al. (2017). New records of long-legged.... Acta Biologica Sibirica, 2017, 3(4), 99-112

DISTRIBUTION. Type locality: Germany: "Hamburg”. Asia (Iran, Israel, Syria, Turkey, Turkmenistan, Siberia), Caucasus (Abkhazia, Armenia, Russia: Adygea, Chechnya, Kabardino-Balkaria, Krasnodar Territory), Europe, North Africa.

Tachytrechus planitarsis Becker, 1907

REFERENCES. Becker, Stein, 1913: 597 (Sistan and Baluchestan: Bampur).

MATERIAL. Lorestan prov.: Horramabad Shahrestan, Sezar River from Bisheh Waterfall to $2 \mathrm{~km}$ downstream,

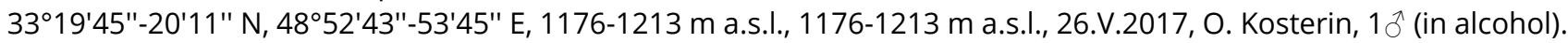

DISTRIBUTION. Type locality: Algeria: Biskra. Palaearctic: Asia (Iran, Israel, Saudi Arabia, Turkmenistan), Spain (Canary Is.), North Africa; Afrotropics (Ethiopia). New for Lorestan province.

REMARK. The species was not collected in Iran since N. Zarudny's (St. Petersburg, Russia) 1898 and 1901 expeditions.

Tachytrechus sogdianus Loew, 1871 (Fig. 13)

MATERIAL. Lorestan prov.: Aligudarz Shahrestan, Golbahar River at Golbahar-e Seikh Miri and Golbahar-e Mohammad

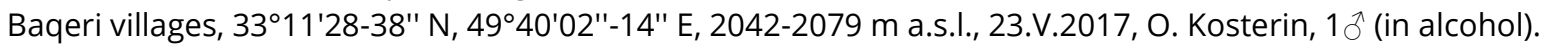

ADDITIONAL MATERIAL EXAMINED. Tajikistan: Sangvor, 10.VI.1977, Zlobin, 1 đ̂. for Iran.

DISTRIBUTION. Type locality: Tajikistan: Zeravshan Valley, Varzaminor. Asia (Kyrgyzstan, Tajikistan, Uzbekistan). New

Teuchophorus Loew, 1857

Teuchophorus sp.

MATERIAL. Lorestan prov.: 22 km S Aligudarz, Golbahar River at Golbahar-e Seikh Miri and Golbahar-e Mohammad

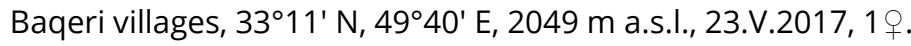

REMARK. First record of the genus for Lorestan province.

Thinophilus Wahlberg, 1844

Thinophilus flavipalpis (Zetterstedt, 1843) (Figs. 8-10)

MATERIAL. Markazi prov.: Arak env., Kavir-e Meighan, Lake S bank, 34099' N, 4949' E, 1672 m a.s.l., 30.V.2017, 1 ô $^{\circ} 1$ ․

DISTRIBUTION. Type locality: Sweden: Gottlandia, Bursviken. Asia (N China, Israel, Kazakhstan, Kyrgyzstan, Mongolia, Syria, Turkey), Caucasus (Azerbaijan, Russia: Krasnodar Territory, Rostov Region), Europe, North Africa; Oriental China. New for Iran.

\section{Discussion}

As a result of 2016-2017 survey conducted in seven provinces, a new material of Dolichopodidae was collected and identified, belonging to 18 genera and 41 (including 4 unnamed) species listed above. Almost all species were gathered along banks of rivers, lakes and other water sources; nevertheless, Medetera species inhabited rather dry places and were taken from tree trunks, stones or even house walls (see Figs. 14-19). The present research gives new records from the Central and North-Eastern Iran, including Dolichopus jaxarticus, Hydrophorus viridis, Medetera diadema, M. lamprostoma, M. roghii, Tachytrechus kowarzi, T. sogdianus and Thinophilus flavipalpis found for the first time in Iran.

Most collected species are widespread across the Palaearctic Region. According to sampling data, three species (S. pallipes, $P$. regalis and T. notatus) have the highest total abundance in at least Markazi and Lorestan provinces. One species (Medetera anjudanica) is a new for science and is described separately (Grichanov, Ahmadi, 2017b), being at present endemic of the Central Iran along with Poecilobothrus lorestanicus. As a result of our investigation, the number of reported dolichopodid species from Iran includes now about 150 species. Fauna of the Markazi province includes 40 identified species, the Lorestan province contains 35, the Tehran province - 17, and the Esfahan province - 9 identified species. We suggest that many more species will be revealed in Iran, if new provinces and localities are investigated with the use of mass trapping methods. The total number of Iranian species can reach to 400-500 species.

\section{Acknowledgements}

The authors are sincerely grateful to Dr. Akram Ahmadi (Arak, Iran) and Dr. Nikita Vikhrev (Moscow, Russia) for the organizing our field trips, as well as to many Iranian friends for their help during the field work.

\section{References}

Ahmadi, A., Gheibi, M., Ostovan, H., Hesami, S., Grichanov, I.Ya. (2016). New records of Dolichopodidae (Diptera) from Central Provinces of Iran. Halteres, 7, 191-196. Available from: http://www.antdiversityindia.com (accessed 15 August 2017).

Ahmadi, A., Gheibi, M., Ostovan, H., Hesami, S., Grichanov, I.Ya. (2017). New records of long-legged flies (Diptera, Dolichopodidae) of Iran. Russian Entomological Journal, 26(1), 65-70. Available from: http://kmkjournals.com/upload/PDF/REj/26/ent26 1065070 Ahmadi et al for Inet.pdf (accessed 15 August 2017).

Becker, Th., Stein, P. (1913). Persische Dipteren von den Expeditionen des Herrn N. Zarudny 1898 und 1901. Ezhegodnik Zoologicheskogo Muzeya Imperatorskoi Akadedmii Nauk, 17(3-4), 503-654. Available from: https://archive.org/details/persischediptere00beck (accessed 15 August 2017). 
Grichanov, I.Ya. et al. (2017). New records of long-legged.... Acta Biologica Sibirica, 2017, 3(4), 99-112

Grichanov, I.Ya. (2007). A checklist and keys to Dolichopodidae (Diptera) of the Caucasus and East Mediterranean. St.Petersburg: VIZR, 1-160 (Plant Protection News Supplements). Available from: http://www.diptera.info/downloads/Grichanov 2007b.pdf (accessed 26 June 2016).

Grichanov, I.Ya. (2016). Review of faunal investigation of predatory flies of the family Dolichopodidae (Diptera) in Iran. Acta Biologica Sibirica, 2(4), 11-14.

Grichanov, I.Ya. (2017). Alphabetic list of generic and specific names of predatory flies of the epifamily Dolichopodoidae (Diptera). 2nd Edition. St.Petersburg: VIZR, 1-563. (Plant Protection News Supplements, N23). Available from: https://archive.org/download/Grichanov2017DolibankSec/Grichanov\%202017\%20Dolibank-sec.pdf (accessed 15 August 2017).

Grichanov, I.Ya., Ahmadi, A. (2016a). On the distribution of Liancalus virens (Scopoli, 1763) (Diptera: Dolichopodidae). Fly Times, 57, 6-10. Available from: http://www.nadsdiptera.org/News/FlyTimes/issue57.pdf (accessed 15 August 2017).

Grichanov, I.Ya., Ahmadi, A. (2016b). A new peculiar species of Poecilobothrus from Iran (Diptera: Dolichopodidae). Caucasian Entomological Bulletin, 12(2), 313-316. Available from: http://www.ssc-ras.ru/en/journal/caucasianentomological-bulletin/electronic c (accessed 15 August 2017).

Grichanov, I.Ya., Ahmadi, A. (2017a). Palaearctic species of the genus Lamprochromus Mik, 1878 (Diptera: Dolichopodidae). Far Eastern Entomologist, 336, 1-12.

Grichanov, I.Ya., Ahmadi, A. (2017b). A new species of Medetera Fischer Von Waldheim, 1819 (Diptera: Dolichopodidae) from Iran. Far Eastern Entomologist, 339, 12-15.

Grichanov, I.Ya., Alikhani, M., Rabieh, M.M. (2010). New data on the distribution of Dolichopodidae (Diptera) in Iran. International Journal of Dipterological Research, 21(3), 195-201.

Grichanov, I.Ya., Negrobov, O.P. (2014). Palaearctic species of the genus Sciapus Zeller (Diptera: Dolichopodidae). St.Petersburg: VIZR, 1-84 (Plant Protection News Supplements, N13). Available from: https://archive.org/details/GrichanovNegrobov2014Sciapus (accessed 15 August 2017).

Kazerani, F., Khaghaninia, S., Grichanov, I.Ya. (2014). The genus Dolichopus Latreille diversity in three different habitats of East Azerbaijan province, with new records for Iran. Arxius de Miscellània Zoològica, 2013, 11, 134-152. Available from: http://amz.museucienciesjournals.cat/files/AMZ vol 112013 pp 134-152 Kazerani et al.pdf (accessed 15 August 2017).

Kazerani, F., Khaghaninia, S., Talebi, A.A., Persson, M., Pollet, M. (2017). Eight new species of Dolichopodinae (Diptera: Dolichopodidae) from northern Iran. Zootaxa, 4242(1), 111-141.

Kazerani, F., Khaghaninia, S., Talebi, A.A., Pollet, M. (2016a). Study of the genus Medetera Fischer von Waldheim, 1819 (Dip., Dolichopodidae) in the forests of northern Iran with 5 new records species for the fauna of Iran. In: Proceedings of 22nd Iranian Plant Protection Congress, 27-30 August 2016. College of Agriculture and Natural Resources, University of Tehran, Karaj, IRAN, 452.

Kazerani, F., Khaghaninia, S., Talebi, A.A., Pollet, M. (2016b). Faunistic study of Rhaphinae Bigot, 1852 and Sympycninae Aldrich, 1905 (Dip., Dolichopodidae) in North and Northwest Iran, with record of 5 new species for the fauna of Iran. In: Proceedings of 22nd Iranian Plant Protection Congress, 27-30 August 2016. College of Agriculture and Natural Resources, University of Tehran, Karaj, IRAN, 455. Available from: https://ippc22.ut.ac.ir/paper?manu=24502 (accessed 15 August 2017).

Khaghaninia, S., Gharajedaghi, Y., Grichanov, I.Ya. (2014). A contribution to the knowledge of the family Dolichopodidae (Diptera) in East Azerbaijan province of Iran. Check List: The Journal of Biodiversity Data, 10(3), 588-593. DOI: http://dx.doi.org/10.15560/10.3.588.

Negrobov, O.P. (1991). Dolichopodidae. In: Soos, A., Papp, L., Oosterbroeck, P. (Eds.). Catalogue of Palaearctic Diptera 7: Dolichopodidae-Platypezidae. Budapest: Akadémiai Kiadó, 1-291.

Negrobov, O.P., Naglis, S. (2016). Palaearctic species of the genus Medetera (Diptera: Dolichopodidae). Zoosystematica Rossica, 25(2), 333-379. Available from: https://www.zin.ru/journals/zsr/content/2016/zr 2016252 Negrobov.pdf (accessed 15 August 2017).

Negrobov, O.P., Selivanova, O.V., Maslova, O.O., Chursina M.A. (2013). Check-list of predatory flies of the family Dolichopodidae (Diptera) in the fauna of Russia. In: Grichanov, I.Ya., Negrobov, O.P. (Eds.). Fauna and taxonomy of Dolichopodidae (Diptera). Collection of papers. St.Petersburg: VIZR RAAS (Plant Protection News Suppl.), 47-93. Available from: https://archive.org/details/GrichanovNegrobovDolichopodidae2013 (accessed 15 August 2017).

Pârvu, C. (1996). Dolichopus siculus stat.n. (Diptera: Dolichopodidae) and some other species not mentioned in Israel [Results of the "Emil Racovita" Speleological Institution of Bucharest - Romania in Israel - 1995]. Travaux du Muséum national d'Histoire naturelle "Grigore Antipa", 36, 279-285. Available from: https://www.travaux.ro/web/pdf/36TMNHNGA279-285.pdf (accessed 15 August 2017).

Tajmiri, P., Kazerani, F., Fathi Seyed, A. (2016). First record of Sciapus longulus (Fallen 1823) (Dip., Dolichopodidae) from Iran. In: Proceedings of 22nd Iranian Plant Protection Congress, 27-30 August 2016. College of Agriculture and Natural Resources, University of Tehran, Karaj, IRAN, 468. 

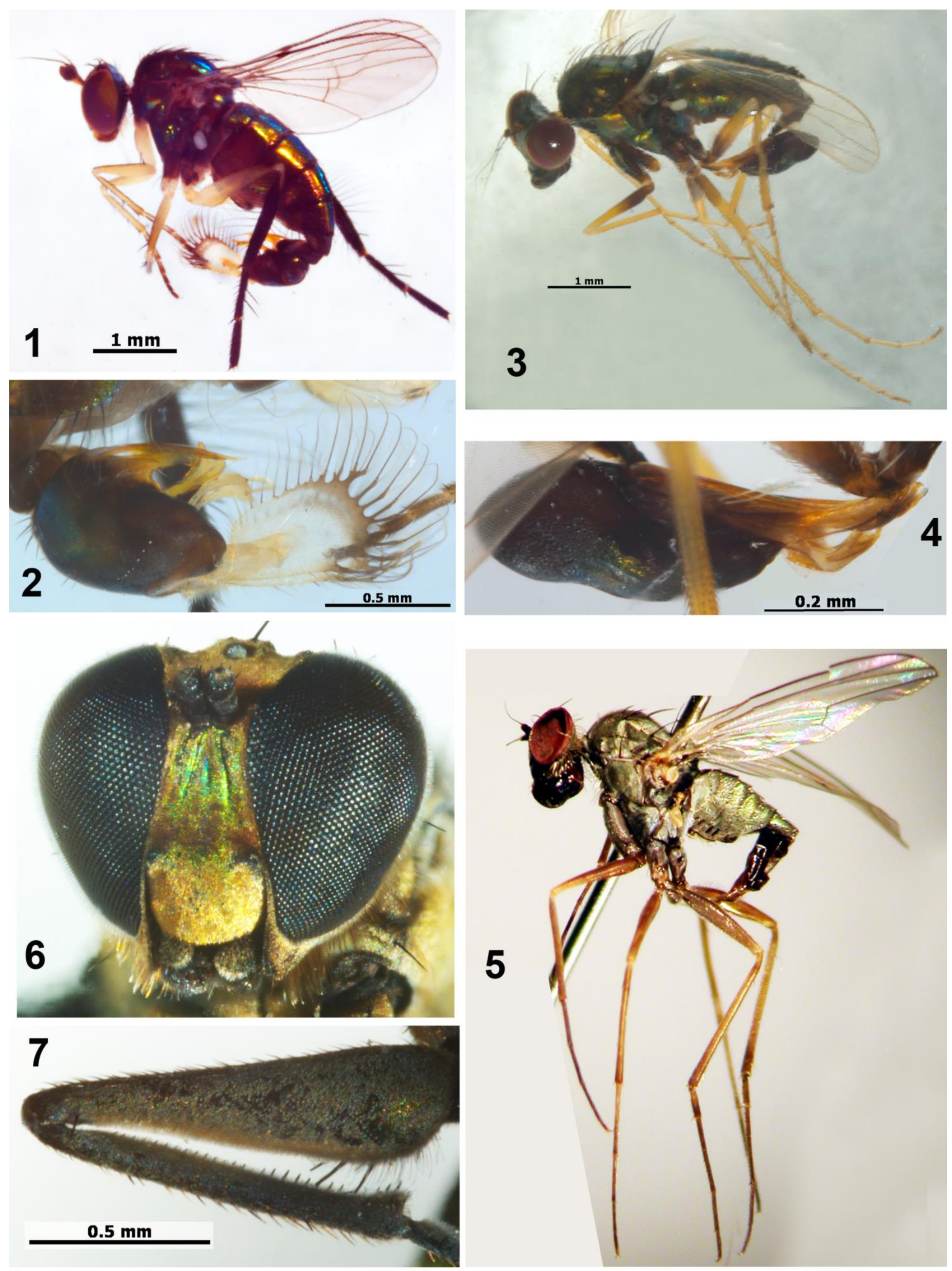

Figs. 1-7. Diagnostic characters of some species firstly found in Iran: Dolichopus jaxarticus Stackelberg, 1927: 1 - male habitus, 2 - hypopygium; Medetera lamprostoma Loew, 1871: 3 - male habitus, 4 - hypopygium; Medetera diadema (Linnaeus, 1767): 5 - male habitus; Hydrophorus viridis (Meigen, 1824): 6 - female head, 7 - fore leg. 

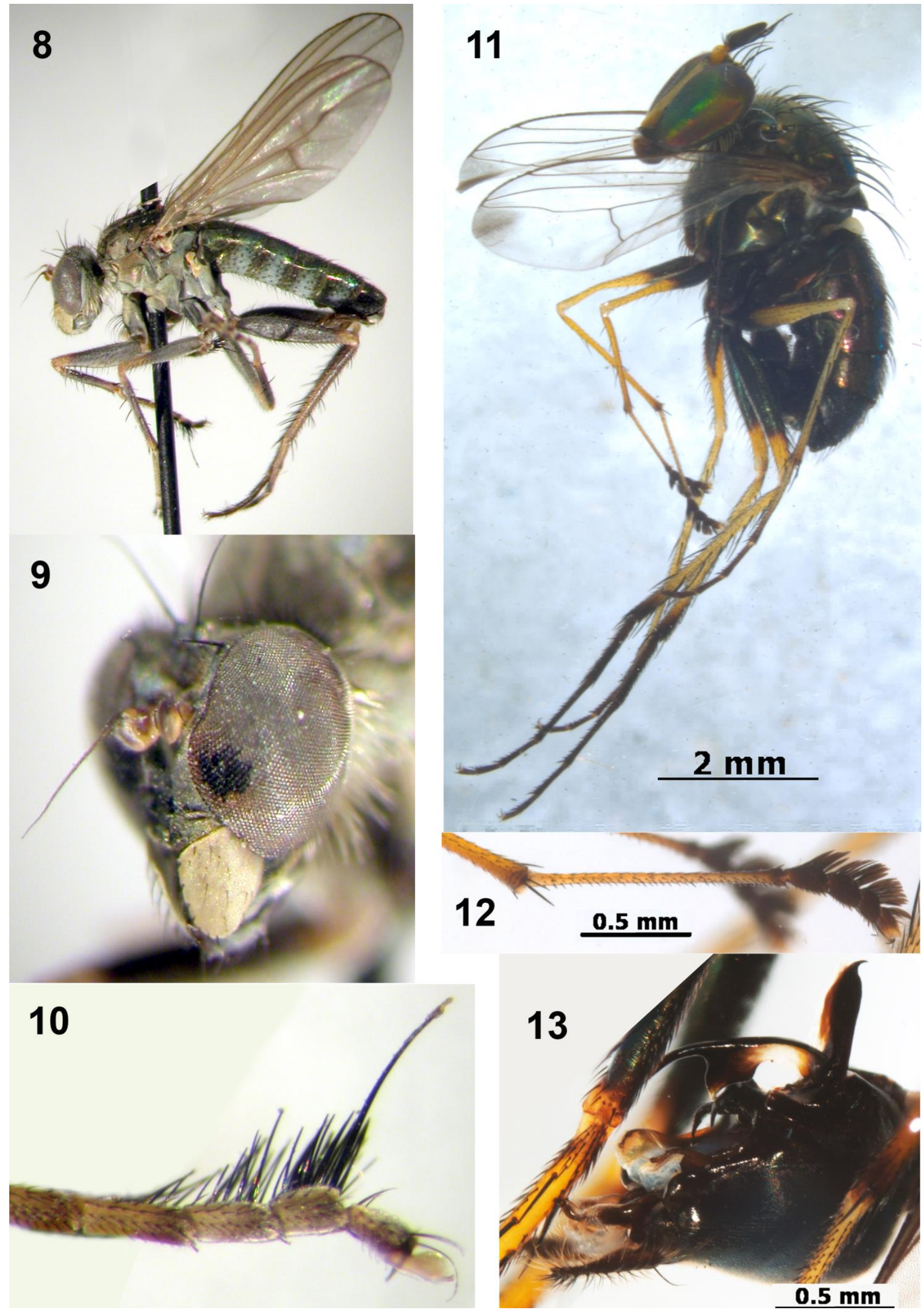

Figs. 8-13. Diagnostic characters of some species firstly found in Iran: Thinophilus flavipalpis (Zetterstedt, 1843): 8 - male habitus, 9 - head, 10 - fore tarsus; Tachytrechus kowarzi Mik, 1864: 11 - male habitus; 12 - fore tarsus; Tachytrechus sogdianus Loew, 1871: 13 - hypopygium. 


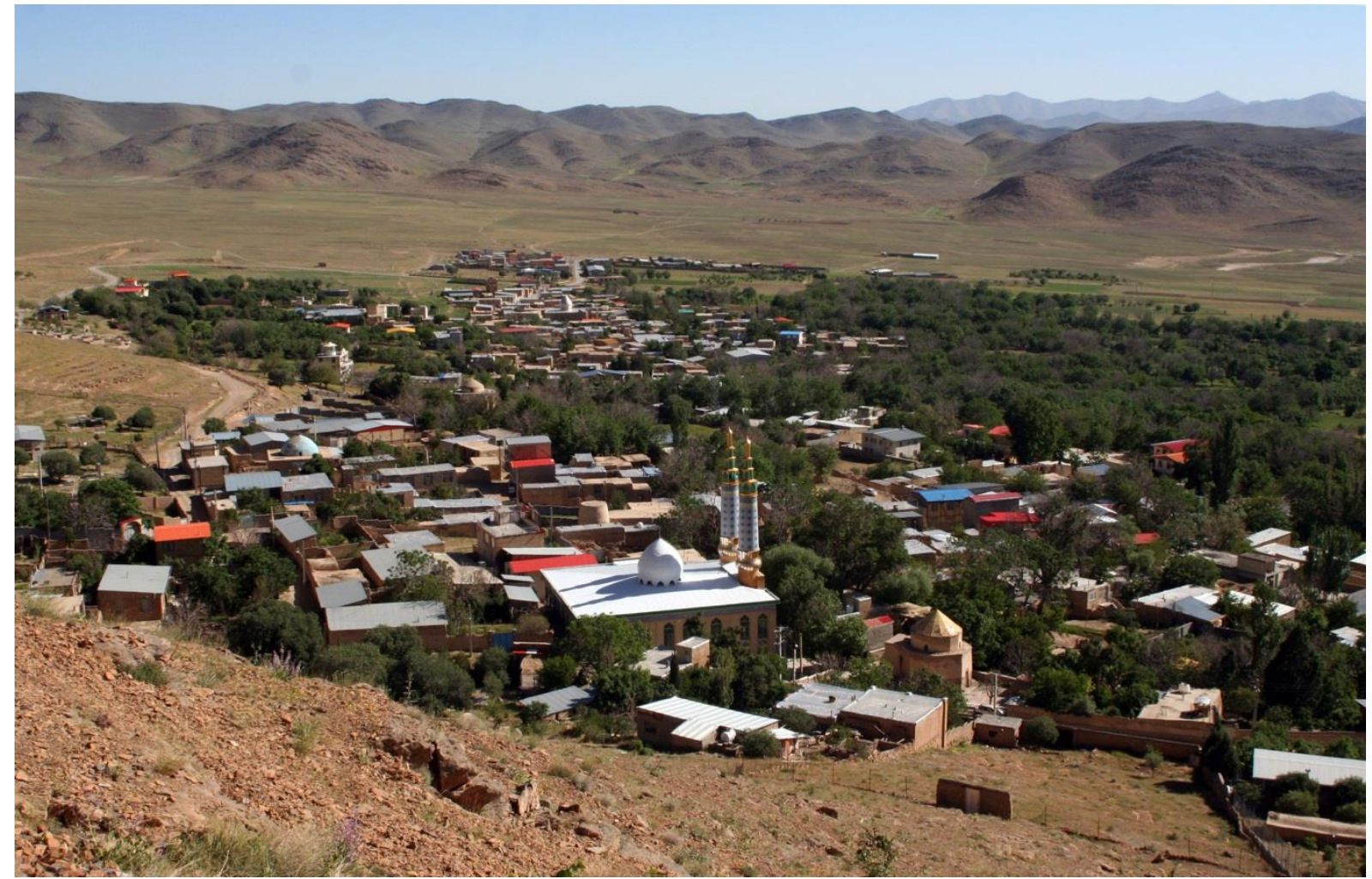

Fig. 14. A historical Iranian village, Anjudan (or Anjedan), where the type series of Medetera anjudanica Grichanov et Ahmadi, 2017 was collected (on a house wall). Markazi province, 22 May 2017

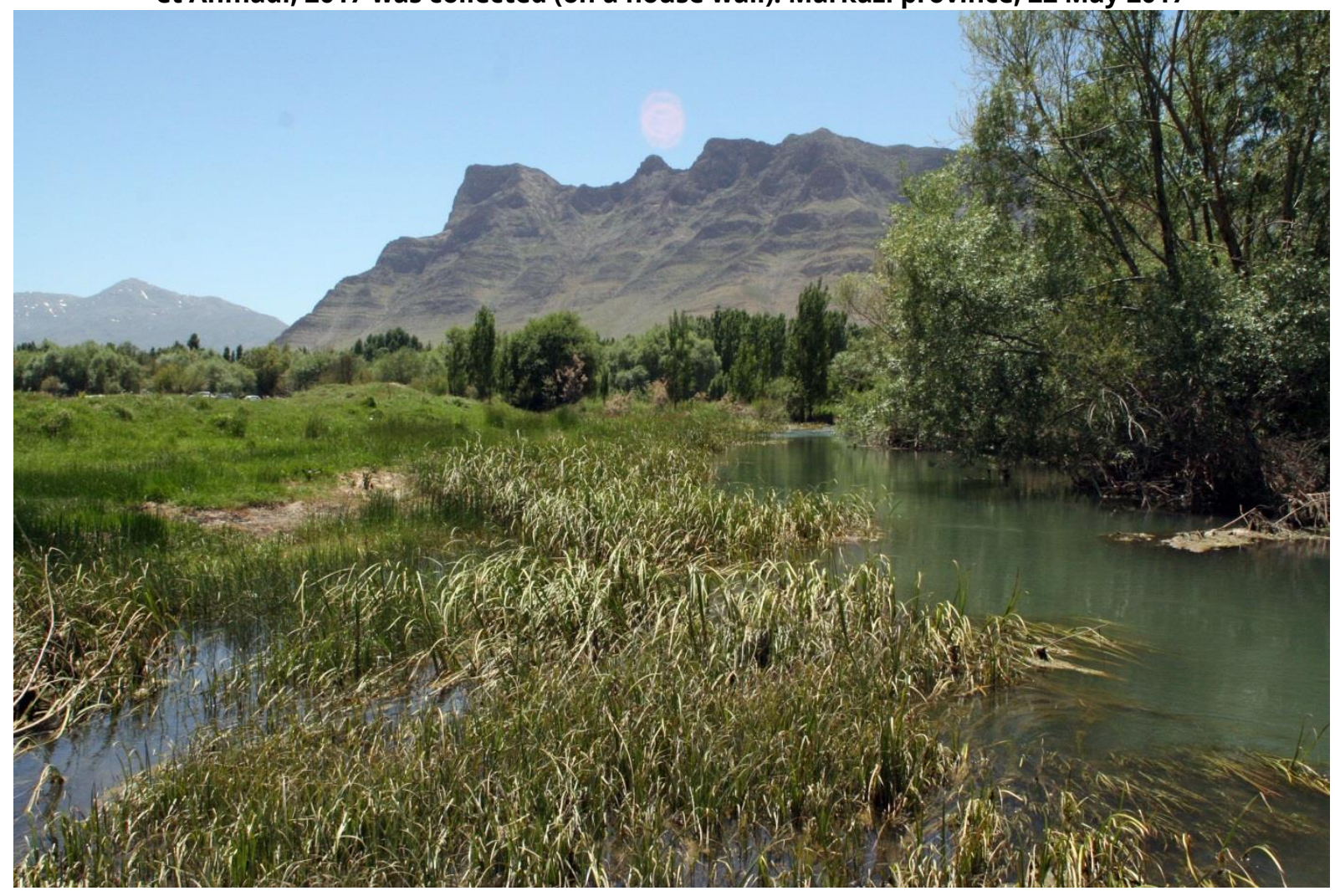

Fig. 15. Garehchay River bank, a habitat for Tachytrechus and Hydrophorus species.

Markazi province, 19 May 2017. 
Grichanov, I.Ya. et al. (2017). New records of long-legged.... Acta Biologica Sibirica, 2017, 3(4), 99-112

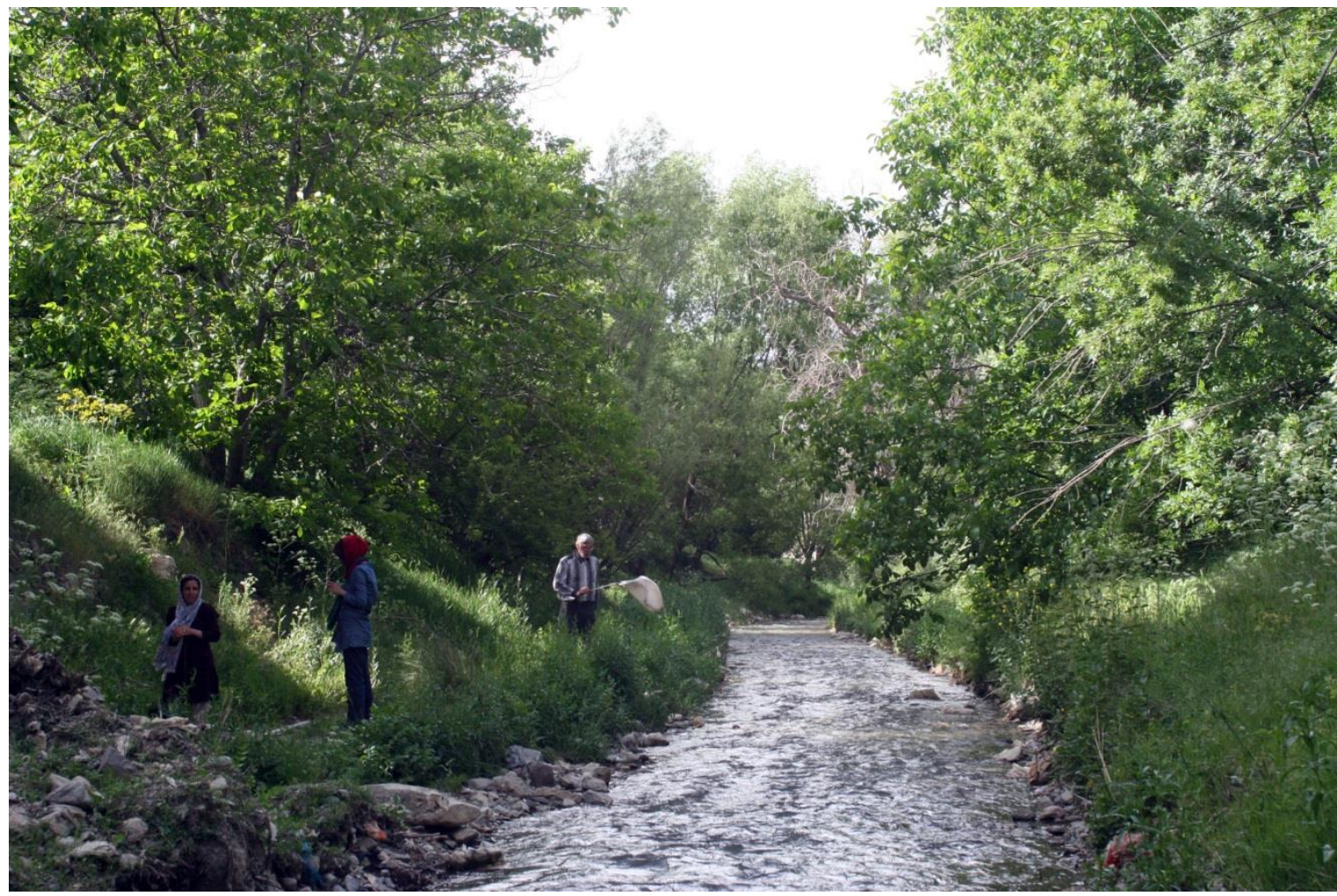

Fig. 16. Ghomrood River, a habitat for Campsicnemus and Syntormon species. Esfahan province, 21 May 2017.

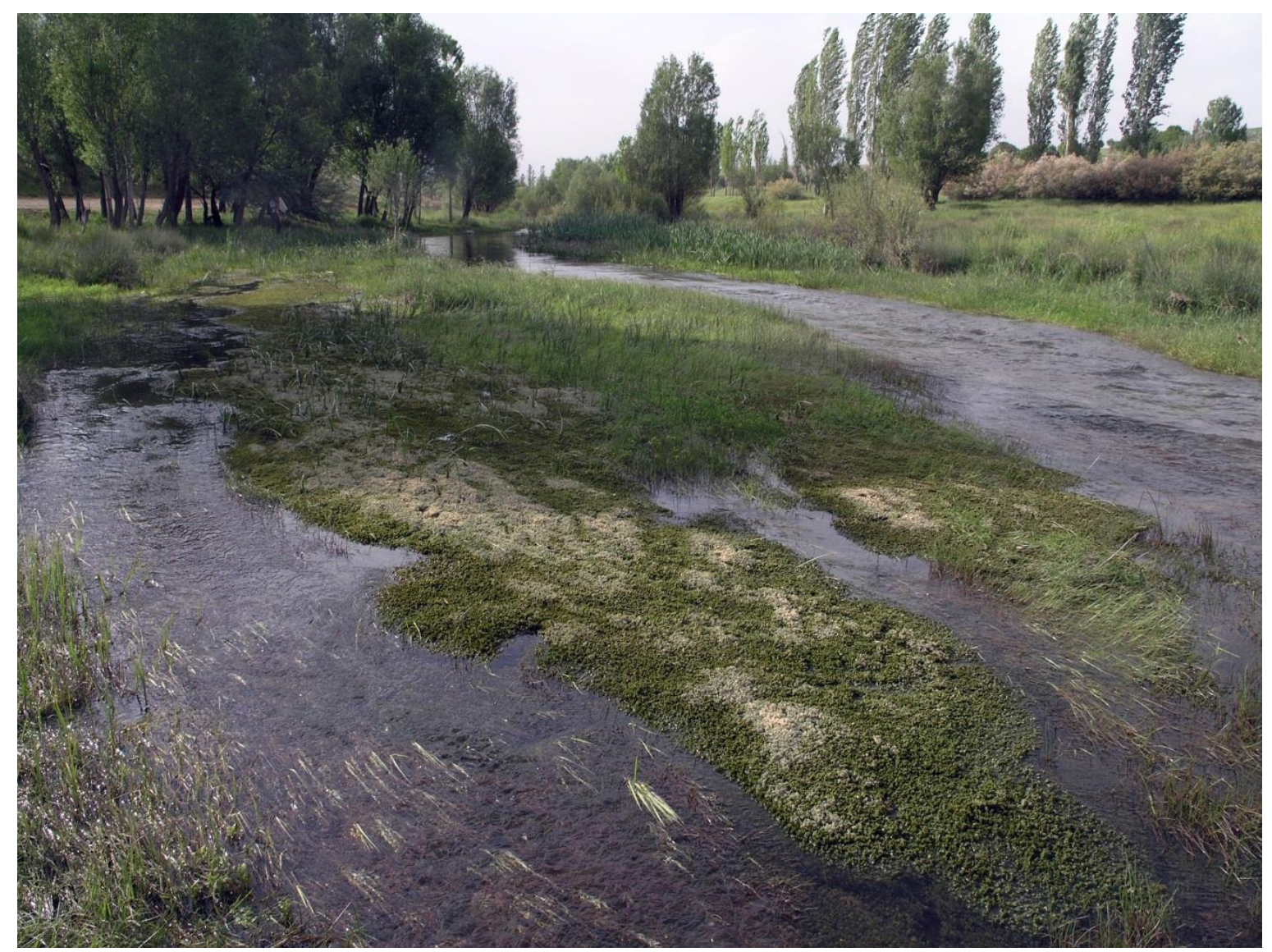

Fig. 17. Hendadar River, a habitat for Campsicnemus, Dolichopus and Syntormon species. Markazi province, 20 May 2017. 


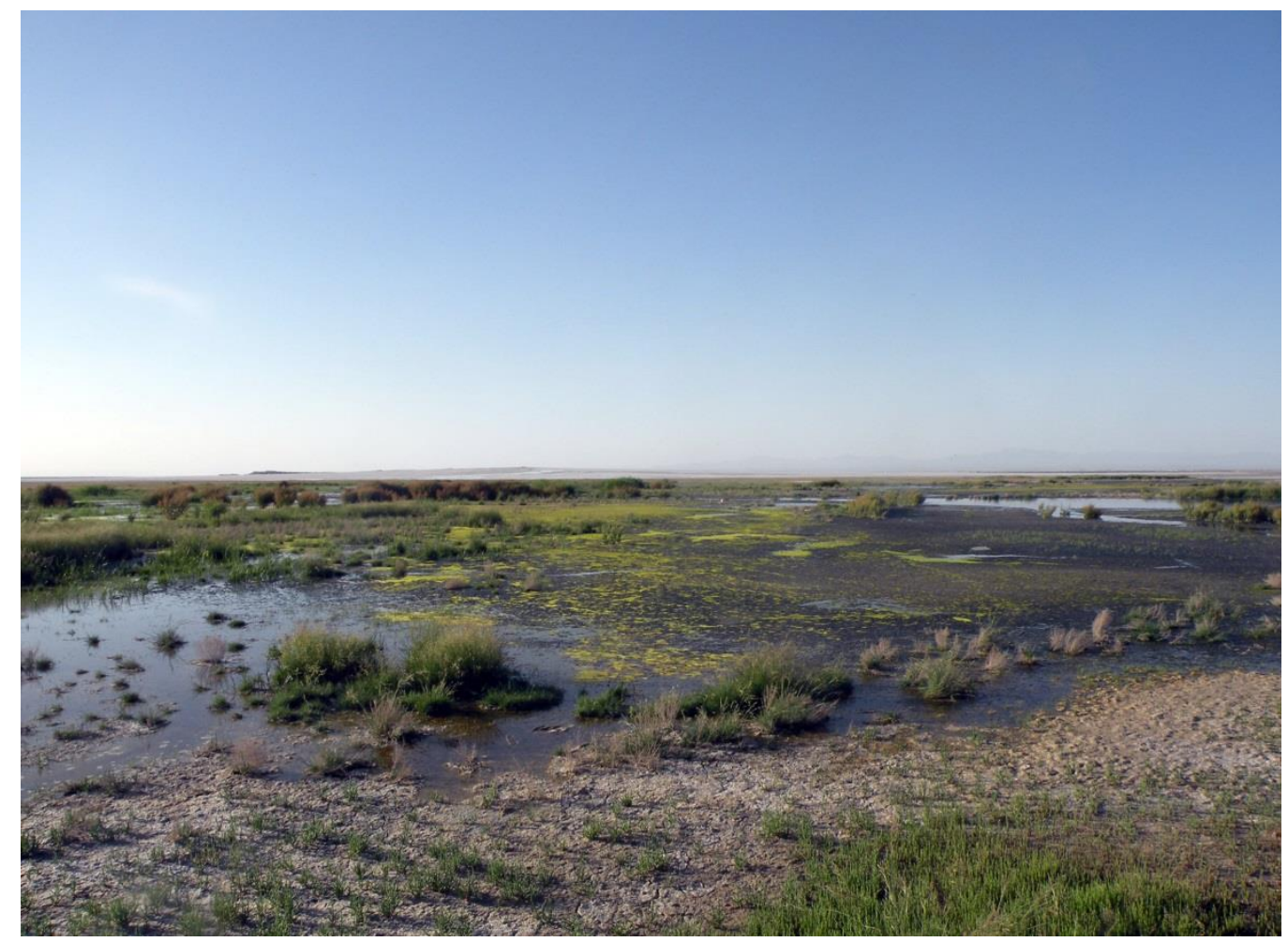

Fig. 18. South bank of Kavir-e Meighan Lake, a habitat for Thinophilus flavipalpis.

Markazi province, 30 May 2017.

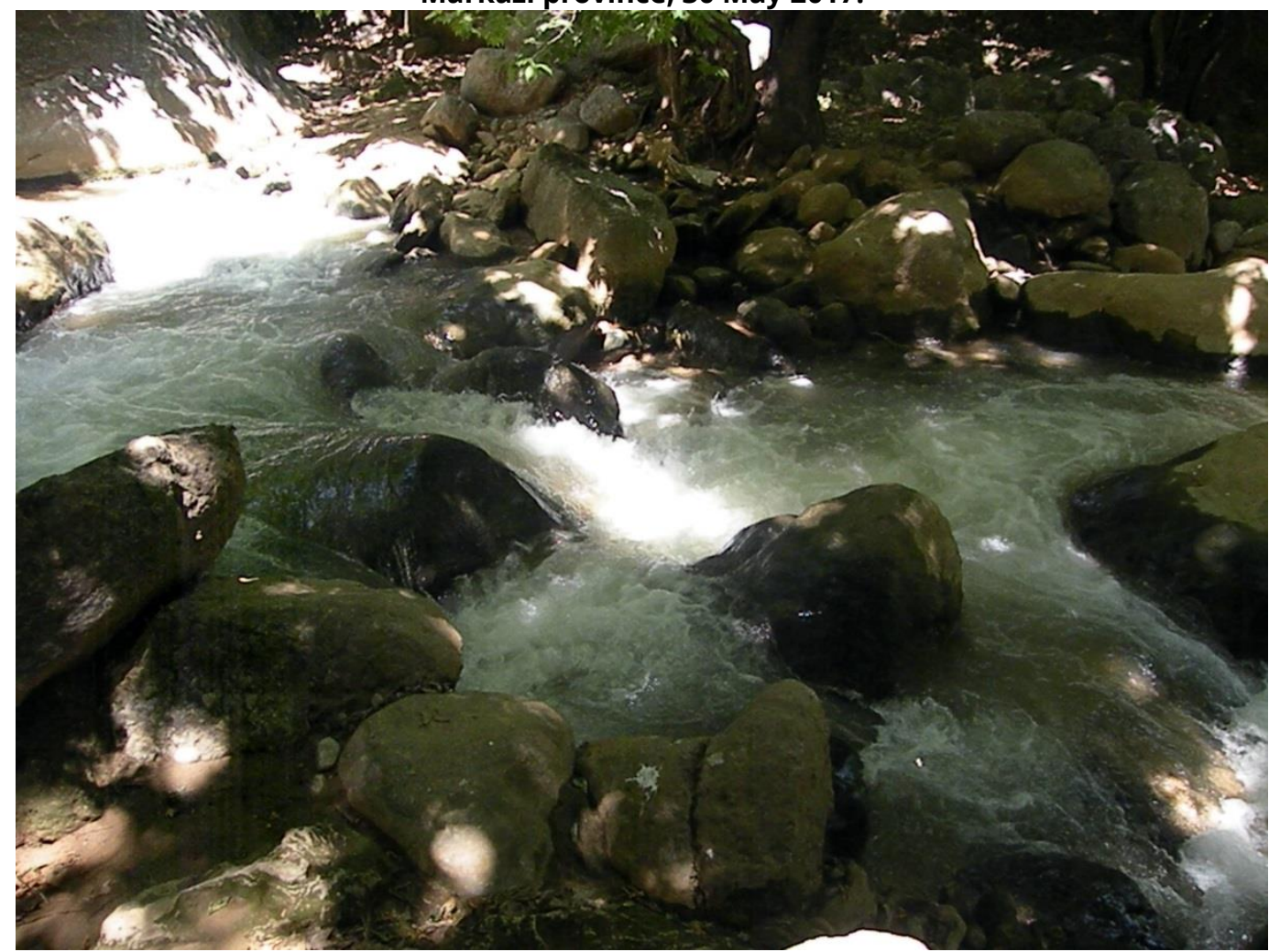

Fig. 19. Sezar River at 2 km downstream of Bisheh Waterfall with wet stones - a resting place for Liancalus virens. Lorestan province, 26 May 2017.

\section{Citation:}

Grichanov, I.Ya., Ahmadi, A., Kosterin, O.E. (2017). New records of long-legged flies (Diptera, Dolichopodidae) from Central and North-Eastern Iran. Acta Biologica Sibirica, 3 (4), 99-112.

Submitted: 25.07.2016. Accepted: 14.09.2017

cross ref http://dx.doi.org/10.14258/abs.v3i4.3636 